\title{
Change Scheduling based on Business Impact Analysis of Change-Related Risk
}

\author{
Thomas Setzer, Member, IEEE, Kamal Bhattacharya, Member, IEEE, and Heiko Ludwig, Member, IEEE,
}

\begin{abstract}
In todays enterprises, the alignment of IT service infrastructures to continuously changing business requirements is a key cost driver, all the more so as most severe service disruptions can be attributed to the introduction of changes into the IT service infrastructure. Change management is a disciplined process for introducing required changes with minimum business impact. Considering the number of business processes in an enterprise and the complexity of the dependency network of processes to invoked services, one of the most pressing problems in change management is the risk-aware prioritization and scheduling of vast numbers of service changes. In this paper we introduce a model for estimating the business impact of operational risk resulting from changes. We determine the business impact based on the number and types of business process instances affected by a change-related outage and quantify the business impact in terms of financial loss. The model takes into account the network of dependencies between processes and services, probabilistic change-related downtime, uncertainty in business process demand, and various infrastructural characteristics. Based on the analytical model, we derive decision models aimed at scheduling single or multiple correlated changes with the lowest expected business impact. The models are evaluated using simulations based on industry data.
\end{abstract}

Index Terms-Business Impact Analysis, Change Management, Change Scheduling, Risk Management, Service Networks, Service Transition Management.

\section{INTRODUCTION}

$\mathbf{T}$ ODAY'S businesses operate in dynamic environments with the need to continuously adapt to changing customer expectations, market trends, technical enhancements, or changes to legislation. These adaptations mostly entail changes to IT services and business processes to drive the alignment of IT with business requirements.

According to recent surveys, uncontrolled changes including flawed risk and impact analysis cause more than $80 \%$ of severe business-critical service disruptions [1], and change management $(\mathrm{CM})$ is frequently one of the first processes included when an enterprise initiates the implementation of IT service management (ITSM). ITSM has received much attention in recent years, as enterprises understand that operating their IT is a large part of their overall operating costs. CM is

Manuscript received January 26, 2009; revised October 7, 2009 and November 30, 2009; accepted December 17, 2009 . The associate editor coordinating the review of this paper and approving it for publication was Prof. Dr. Heinz-Gerd Hegering.

T. Setzer is with the Department of Informatics, Technische Universität München, 85748 Garching, Germany, phone: +49 17624037 295; email: thomas.setzer@in.tum.de (corresponding author).

K. Bhattacharya and H. Ludwig are with the IBM T.J. Watson Research Center, Business-driven IT Management Research Group, 19 Skyline Dr., Hawthorne, NY-10532, USA; email: \{kamalb, hludwig\}@us.ibm.com.

Digital Object Identifier an ITSM process for introducing required changes into the IT environment, with the underlying objective of minimizing disruptions to the business services as a result of performing IT changes.

Publicly available best-practice ITSM frameworks such as the IT Infrastructure Library (ITIL) [2] define a high-level reference $\mathrm{CM}$ process including several activities like change initiation, where a request for change (RFC) describing the required change is submitted, change filtering, priority allocation, categorization, planning, testing, scheduling, fulfillment, and review.

According to a survey with IT change managers in 2006, currently the most pressing problems in CM are the risk-aware classification and the planning and scheduling of - partly urgent - changes, as performing IT changes generally entails a risk of resulting service downtime [3].

The problem with classifying changes is that IT practitioners have little visibility of business risk and the impact of changes on customers. To make as much information as possible available to all the stakeholders, ITIL recommends the creation of a change advisory board (CAB), typically made up of decision-makers from IT operations and business units who meet weekly to review change requests, evaluate risks, identify impacts, etc.

Major changes - entailing high risk to the business - must be analyzed and approved by the $\mathrm{CAB}$, from a technical as well as from a business point of view before they schedule the ones they approve.

The distinction between major and minor changes is vital, as IT departments of medium and large organizations usually need to process up to 400 RFCs per week [3], [4]. This sheer volume of RFCs makes CM very challenging, makes $\mathrm{CAB}$ meetings tedious, and consume a great deal of time. Besides the ever-increasing number of changes, the constantly growing complexity of IT infrastructures further complicates the problem. Furthermore, classifying changes in modern IT environments is a challenging problem because of the multiple interdependencies and uncertainties to manage. Consider the following example, illustrated in Fig. 1, which we find in many companies. A business application runs several customer relationship management (CRM) processes, from lead generation to sales order generation. The application itself is hosted on one or more physical resources and has dependencies to other applications (or services) and infrastructural components. The scenario on the left-hand side shows a pictorial of a Tivoli Change and Configuration Management Database (CCMDB) discovery [5], where Application A uses Application B. Downtime of Application B means an impact on Application A. This 

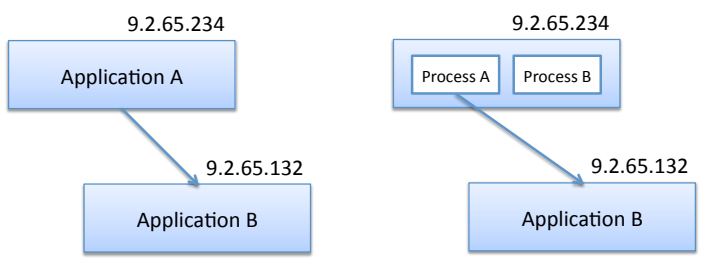

Fig. 1: Business Process Application Dependencies

view however is not sufficient as an organization managing the business processes will alert business users that the CRM application will be unavailable, which could lead to unfulfilled sales orders.

The picture on the right-hand side illustrates the more realistic, fine-grained scenario where Application A is hosting two processes, e.g. lead generation and sales order generation. The actual downtime of Application B may only lead to the unavailability of lead generation but not sales order generation (which in the CRM context is a much lower risk). Furthermore, based on the fact that the affected lead generation may be a longer-running business process, one can imagine that only a subset of all running instances will be affected depending on the state of each instance. How many instances of a particular process are affected further depends on the business process demand while implementing the change, which is generally not known a-priori but has to be approximated by means of forecasting techniques. However, the longer the downtime for a given service is, the more likely it is to experience business value attrition, for example due to SLA violations and associated penalties.

In general, in today's enterprises an increasing number of core business processes run in an automated or semiautomated way on distributed transaction processing (DTP) systems. These systems typically have a large-scale and complex architecture that has grown over time.

DTP systems of medium or large companies typically consist of tens of processes that are composed of hundreds of heterogeneous, distributed IT services. Furthermore, modern IT service infrastructures are continuously transformed towards virtualized, shared hardware pools and Service-Oriented Architectures (SOA) [6].

In such environments, applications as well as hardware functionality can be viewed as services shared in a larger value network and invoked in the context of various processes.

Services can be described using standards such as the Interface Description Language (IDL) or the Web Service Description Language (WSDL), defining for example the interfaces of services offered as Web services, and invoked via a suitable Internet protocol.

We distinguish between different types of services. An atomic service in our definition is a service with a welldefined transaction boundary that provides a simple single operation (e.g. generate IP or assignServerName). A business process is executed by invoking atomic services, other services that may be composed of atomic services (e.g. short-running transactions), or other business processes. Furthermore, each atomic service is executed on an IT resource.

Considering the number of business processes in an enterprise and the complexity of the dependency network of processes to invoked services, managing such an infrastructure is a highly complex task. Changes in this kind of environment pose exceptionally high risks as the impact of failures is likely to be business-critical as many business processes might depend on a service. Seemingly routine tasks, such as moving a server or reconfiguring a router, might result in unplanned downtime of core business processes. Therefore, efficient and reliable $\mathrm{CM}$ aimed at continuous service delivery considering the dependency chains is essential. We believe that CM should take this level of detail into account to determine and minimize the risk of downtime for business value generating services.

Whereas business process automation and management has been a dominant topic in information systems research in recent years, surprisingly little has been published on business impact and risk analysis of transitions of services involved in business processes.

In this paper we focus on how to schedule service changes in complex service-oriented enterprise information systems in order to minimize the business impact of potential changerelated outage of uncertain duration. By business impact we mean the monetization of change-related impacts on dependent, active business process instances. A solution to this problem is required to automatically consider business process demand forecasting as well as downtime estimation and its uncertainty, as well as the dependency network from a business process down to affected resources, applications, or other services required by business processes.

A decision model is then required to allow organizations the scheduling of single or multiple correlated changes with the lowest expected overall costs.

In the remainder of this paper we propose an analytical model to monetize the estimated business impact of changes and develop a change scheduling decision model based on the analytical model.

This paper is structured as follows. In section II we review related work in this field. Section III discusses techniques to estimate and quantify operational risks of service transitions and how to map these risks to financial loss. Subsequently, in section IV we introduce a deterministic decision model and probabilistic extensions to determine efficient change schedules in different business scenarios with steady and volatile process demand. We further provide model extensions to take into account change correlations and other sources of risk like violating change windows. In section $\mathrm{V}$ we describe an approach to refine the schedule over time by updating demand forecasts and exploiting more reliable information of process demand behavior and process state that becomes available over time. In section VI we evaluate the models by conducting simulations and we present and discuss simulation outcomes. Section VII offers a brief summary and discusses future working plans.

\section{RELATED WORK}

In this section we review previous work in IT change risk management and change scheduling related to the work 
described in this paper. This paper is an extended version of a conference paper published in the proceedings of the IEEE Network Operations and Management Symposium 2008 [7]. The novel contributions of this paper beyond those of the work described in the conference version are indicated at the end of this section.

The definition of risk itself varies broadly according to the specific domain one looks at. In our work we consider risk as uncertainty of outcome', as it is the most general definition of risk [8]. In our case, the outcome is changerelated cost materialized as financial loss, as we are interested in the business impact of changes. To analyze the impact, i.e., resulting costs for the business, we draw on a two-stage approach; first we scan for possible outcomes and quantify these in terms of monetary consequences, and second, we weight these outcomes by their probabilities. This approach is known as probabilistic risk analysis, as introduced in [9].

In IT change management, most approaches found in the literature, like perceived risk approaches, allow for risk analyses that cannot be directly mapped to business or financial impact, or provide general guidance to change management considering associated risk.

Publicly available best-practice ITSM frameworks and standards such as the IT Infrastructure Library (ITIL) [10] or Control OBjectives for Information, and related technology (COBIT) [11], provide guidance on how to perform service management tasks and are validated across a diverse set of environments and situations. Managing service changes or transitions with respect to associated risks has recently become a major focus of these frameworks. For example, the Office of Government Commerce (OGC) released in the latest ITIL version 3.0 a guideline on how to manage service transitions efficiently, in particular with regard to associated risks [8]. However, ITIL and related best-practice frameworks provide high-level guidance for tasks like managing a change, but do not provide guidance on how to do the actual change management implementation, e.g., on how to quantify, and manage change-related risks in a particular business environment.

Some commercial tools and dashboards are available to assist in managing and scheduling changes, but do not directly map risk to expected financial impact [12]-[15].

Scheduling is a field that has received a lot of attention in the past decades. Numerous scheduling problems have been studied and many solution methods have been used [16]. In particular, resource constraint scheduling problems have been well studied [17]. Surprisingly little has been published on scheduling IT service changes, in particular considering associated risk.

Keller and Hellerstein present the CHAnge Management with Planning and Scheduling (CHAMPS) system to automate steps in the execution of changes. The authors propose decision models to solve different scheduling problems like maximizing the number of changes or minimizing the costs associated with change-related downtime. The authors assume knowledge of cost functions for performing a change at time $t$, while we focus on how to derive cost functions from changerelated downtime risk and uncertain process demand [18].

Goolsbey presents an approach to qualitatively evaluate IT change risk, but does not quantitatively analyze risk [19].

Closest in spirit to our work are the works by Rebouas et al., Trastour et al., and Sauve et al. They address the problem of scheduling changes by minimizing the financial loss imposed by SLA violations. The authors explicitly consider uncertainty in change implementation durations and costs resulting from exceeding change windows [20]-[22]. Again, for minimizing financial loss, costs for performing a change at time $t$ are assumed to be known, while our focus is on how to derive these costs based on estimating the impact on dependent business processes.

Our work serves to fill the gap in work addressing the quantification of service change-related risk to active and dependent business processes, enabling the scheduling of service changes with minimum impact on the business.

In the published conference version we focused on analyzing the business impact of a change-related service's outage on dependent, short-running business process instances. We considered smooth business process demand scenarios and we assumed to have fairly reliable demand forecasts.

In this journal version we introduce and evaluate a model for scheduling multiple changes in the presence of volatile process demand for short-running as well as for more infrequent longer-running business transactions. We further consider uncertain and volatile process demand behavior. We extended the formulations of the conference version to allow for the controlling and adjusting of scheduling plans over time, explicitly considering the stochastic nature of future demand and service downtime duration. Furthermore, we take into account the progress (state) of long-running processes and we conducted more exhaustive sets of simulations based on data of a telecommunication provider's DTP system.

\section{SERVICE TRANSITION AND ASSOCIATED RISK TO BUSINESS PROCESSES}

The goal of service transition management is to deploy new service releases into the production environment with minimum impact on the business. Implementing a change is inherently coupled with potential downtime of one or more services, and risk to the business.

We distinguish between unexpected and expected downtime. In the case of unexpected downtime, mostly there is no change-related downtime but inherently a certain probability of an outage of unknown length. An example might be the hot deployment of an update that fails in $0.5 \%$ of cases and the previous state needs to be restored and reconfigured manually, which takes between five minutes and thirty minutes time where a service is unavailable. Considering expected downtime (e.g., average time period required for cable unplugging and plugging when replacing a switch), an estimator for the average downtime duration might be known beforehand, but downtimes deviates from change to change, even though possibly within tight boundaries.

From a statistics point of view, in both cases change-related downtime is a random variable following a certain probability distribution.

As described in section I, service transition in ServiceOriented DTP systems is coupled with exceptionally high risk 
and complexity, as there are multiple interdependencies and uncertainties to manage and many business processes might depend on a service. It is therefore imperative that IT organizations analyze and eliminate as many change-related risk as possible by increasing the visibility of IT interdependencies. Therefore, a clear picture and a formal description of the business process and service dependency structure is vital at the beginning.

\section{A. Dependency structure}

Existing process and service definitions, for example formalized in the Web Service Business Process Execution Language (WS-BPEL) or the Business Process Modeling Notation (BPMN), can be used to derive parts of the dependency structure, although such definitions typically contain only services with a certain level of abstraction from hardware functionality and do not cover the whole dependency structure. Furthermore, they address many aspects not of immediate interest here.

To derive dependency structures, ITIL proposes the usage of a configuration management database (CMDB). In terms of ITIL, so-called configuration items (CI) should be created and catalogued in a CMDB that acts as a repository for CIs and their relationships. A CI is defined as any identifiable item that supports business services or processes, from hardware components to business processes themselves. In the spirit of SOA, we can map every service to a CI and vice versa.

We will now introduce a notation that is used throughout this paper to formalize process and service dependencies. The model parameters can be derived from information in an existing CMDB or in business process models as long as the CIs and their relationships are described.

Let $S:=I \cup J \cup K$ be the set of all CIs, where the subsets $I, J$, and $K$ are mutually disjoint. Let $I$ be the subset of $|I|$ different business process types $i$. Let $J$ denote the subset of $|J|$ second layer service definitions $j$, describing an aggregated service on the layer below the business process layer. This layer represents automated workflows that merely string together several atomic services $k$ where $K$ denotes the set of $|K|$ atomic services. Given all relations $u_{a b}$ modeling a dependency with either $(a \in I$ and $b \in J)$ or $(a \in J$ and $b \in K)$, the full dependency graph of the three-layer system is derived (modeling four or more layers is straightforward). A relationships $u_{i j}$ indicates that a particular business process type $i$ uses workflow $j$ in process step $u_{i j}$. We set $u_{i j}:=0$ if a business process $i$ definition does not include an aggregated service $j$. In the same manner we model the dependencies of lower-level services by assigning atomic service types $k$ to aggregated services flows $j$ correspondingly by setting $u_{j k}$ to the step in $j$ 's definition. Fig. 2 illustrates the resulting dependency model. This dependency model provides the means of automatically deriving which higher-level services and business processes might be affected by a specific service downtime. However, to estimate the business impact, additional information is required, for example how costs can be assigned to a process disruption or delay, or how many instances of business processes will be disrupted or delayed.

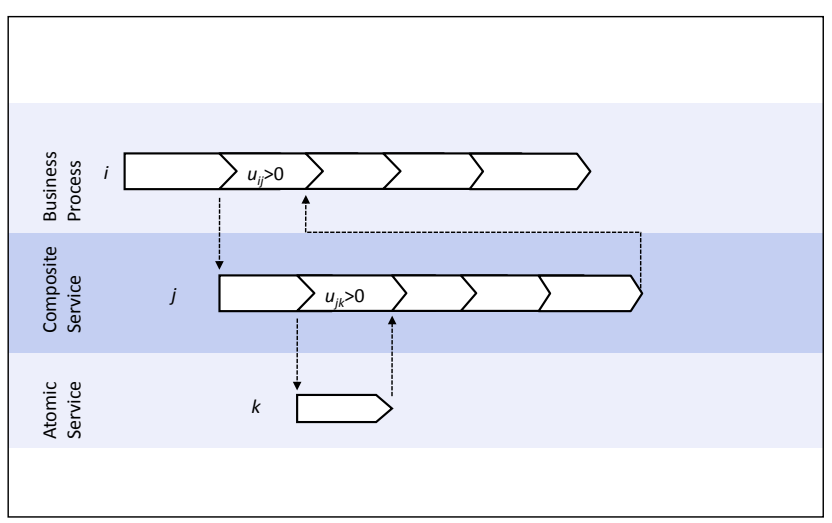

Fig. 2: Three-Layer Service Dependency Model

\section{B. Cost of a business process disruption or delay}

The business impact in terms of costs associated with business process disruptions or delays depends strongly on the business an enterprise is running, the type of a business process, the time of day, and the day of week. Prior work on estimating these costs mostly measured the direct loss of revenue for online companies (Web shops, online travel agencies, etc.) or other services that cannot possibly function if services are down [23]. For lost revenue that is directly assignable to workload lost during a service or process failure, Walsh et al. propose simply multiplying the amount of workload that is expected to be lost from the simulated element failures by a cost-per-unit ratio [24].

However, Patterson - among others - stated that sales processes of such businesses are not the only ones that lose income if the availability or performance of processes is affected [25]. In Patterson's model, cost is a function of lost revenue and lost productivity, as there is for example a loss to a company in wasting employees' time if they cannot get their work done. There are more, although less obvious, costs that can be included in such a business impact model, such as penalty payments imposed for missed deadlines, late fees for untimely delivery, costs of opportunity of lost future revenue, or erosion of company and/or IT department reputations.

Some models like Hewlett Packard's model of downtime costs include the calculation of estimates of significant other charges as mentioned above on an application-by-application basis without considering service dependencies [26].

Total costs need to be considered by an organization when services or whole business processes are outsourced to an IT service provider because outsourcing typically involves the transfer of responsibility as well. The higher the estimated loss resulting from delayed or disrupted business processes will be, the more incentives are advised for a service supplier to mitigate downtime that may lead to disruption or delay.

A common business practice is therefore the inclusion of Service Level Agreements (SLA) in contracts between an organization and a supplier, involving financial penalties if agreements are missed. Therefore, from a service provider's point of view, the function to map delays or disruptions of business processes into costs is explicitly formalized in SLAs, 
typically including a process' maximum response or execution time $L_{i, \max }$ and the definition of penalties $p_{i}$ to pay on SLA violations. Depending on individual SLAs, penalties are paid per maximum response time violation, if the number of service level violations during a given time span exceeds a defined threshold value, or other agreements. Note that in today's profit center-oriented enterprises, SLAs are typically defined between a company's departments and its central IT department as well. In our work we assume SLAs to be defined beforehand and we aim at minimizing total SLA-based penalty payments with our change-scheduling model.

\section{Process demand and process state}

The number of affected business process instances depends on the number of active instances and their states during a change-related service downtime. Business forecasting techniques are used to estimate the demand for a certain business process during a particular period of time. In our conceptual model, for the sake of computational efficiency we divide time into small discrete time intervals $t(t=1, \ldots, T)$, wherein we assume fixed demand levels. Let $D_{i}$ be $i$ 's overall demand profile (i.e., the demand probability distributions $D_{i t}$ of all considered time intervals). Demand forecasts for time interval $t, d_{i t}$, can be derived for example by setting $d_{i t}$ to $D_{i t}$ 's mean value or to a certain percentile of $D_{i t}$ 's distribution.

Let $\Delta t_{j}^{d}$ indicate service $j$ 's change-related downtime length in time intervals. Multiplying the number of expected instances of all process types $i$ with $u_{i j}\left(u_{i j}>0\right)$ during $\Delta t_{j}^{d}$ with associated penalties overrates change-related costs, as not all running process instances will necessarily be affected.

Consider business process instances that already passed the step invoking the changed service. These instances will not be affected, nor is there an impact on running process instances that will execute the changed service after its downtime, once the service is again available. Furthermore, processes and services might be queued, with a certain chance to rollback and execute affected process instances still within time.

A procedure is described below to estimate the number of SLA violations if queuing is not possible. Later we extend our discussion by including queuing processes and services. We start out with a deterministic model, assuming reliable knowledge of process demand and change-related downtime, followed by introducing a probabilistic model to account for uncertainty in both demand and downtime.

\section{Deterministic business impact model for smooth demand}

Consider an RFC for service $j$ with a downtime $\Delta t_{j}^{d}-$ known a priori - starting at $j$ 's change time $t_{j}$. For estimating the business impact we approximate the fractions of the demand per dependent process type $i, d_{i j t}^{p}$ that will be penalized (please note that the penalized demand is labeled with subscript $p$ ) because of $j$ 's outage. Multiplying this number with a penalty payment $p_{i}$, associated with each SLA violation of a business process type $i$, the costs $c_{j t}$ of $j$ 's outage are given in (1).

$$
c_{j t}=\sum_{i} p_{i} d_{i j t}^{p}
$$

To estimate $d_{i j t}^{p}$ we proceed as follows. Because in our basic model we assume that services cannot be queued, all process instances executing $j$ during time period $\left[t_{j}, t_{j}+\Delta t_{j}^{d}\right]$ are automatically disrupted. We assume equal arrival rates of business process requests per time interval (principle of indifference). This assumption is tight as long as the forecasting time intervals (timeslots) are kept small. Of interest is the demand for a business process $i$ not only during the changerelated downtime $\left[t_{j}, t_{j}+\Delta t_{j}^{d}\right]$ but also before $t_{j}$, as running process instances starting before $t_{j}$ might reach service $j$ during $\left[t_{j}, t_{j}+\Delta t_{j}^{d}\right]$. Let $L_{j}^{s}$ be the execution time of service $j$ and let $L_{i}$ be $i$ 's overall process execution duration. We assume deterministic values for $L_{j}^{s}$ and $L_{i}$, where $L_{i}$ equals the sum of execution times of services invoked by $i$.

As in our basic model we assume smooth business process demand profiles around $t_{j}$, the number of active $i$-instances equals $d_{i t} \cdot L_{i}$, the demand for $i$ per timeslot multiplied by $i$ 's duration. Multiplying this value with the probability $L_{j}^{s} / L_{i}$ that an $i$-instance executes $j$ at $t_{j}$, gives us an estimate for the number of active $i$-instances immediately disrupted in $t_{j}$. The number of $i$-instances reaching $j$ in $\left[t_{j}, t_{j}+\Delta t_{j}^{d}\right]$ is - on average $-d_{i t} \cdot \Delta t_{j}^{d}$, the process demands per timeslot in $t_{j}$ multiplied by the downtime duration. Thus, the total expected cost of a process's SLA violations can be estimated using (2).

$$
c_{j t}=\sum_{i} p_{i} d_{i t}\left(L_{j}^{s}+\Delta t_{j}^{d}\right)
$$

This cost approximation is appropriate in scenarios with rather steady demand in $\left[t_{j}-\max \left\{L_{i}, \Delta t_{j}^{d}\right\}, t_{j}+\max \left\{L_{i}, \Delta t_{j}^{\text {down }}\right\}\right]$ where the sum of business process instances starting in a timeslot almost equals the sum of instances finished in a timeslot. Here, the step at which a business process uses service $j$ is irrelevant for our calculation. Furthermore, the model assumes that services cannot be queued and continued at a later time.

\section{E. Impact of queuing}

We will now look at the estimated cost of changing $j$ if queuing is supported. If queuing is supported, not all business process instances that execute $j$ during $\left[t_{j}, t_{j}+\Delta t_{j}^{d}\right]$ are automatically disrupted, as process instances may roll back and re-execute $j$ after $t_{j}+\Delta t_{j}^{d}$. Let $L_{i}^{\max }$ denote $i$ 's maximum execution time as defined in an SLA, and let $b_{i}$ $\left(b_{i}:=L_{i}^{\max }-L_{i}\right)$ represent a time buffer calculated as the difference between the maximum and the regular execution time of a process $i$ if all required services are available. Obviously, if $b_{i} \leq \Delta t_{j}^{d}$, all considered $i$-instances will exceed $L_{i}^{\max }$, and if $\left(\Delta t_{j}^{d}+L_{j}^{s}\right)<b_{i}$, no $i$-instance will be delayed or disrupted. If $\left(\Delta t_{j}^{d}<b_{i}<\Delta t_{j}^{d}+L_{j}^{s}\right)$, the percentage of iinstances successfully delivered in time after a rollback and re-execution of $j$ is given in (3).

$$
\frac{b_{i}-\Delta t_{j}^{d}}{L_{j}^{s}}
$$

If $b_{i}>\Delta t_{j}^{d}$, all running $i$-instances invoking $j$ in $\left[t_{j}, t_{j}+\Delta t_{j}^{d}\right]$ are deliverable in time. If $b_{i}<\Delta t_{j}^{d}$, the rate of successfully 
delivered $i$-instances invoking $j$ in $\left[t_{j}, t_{j}+\Delta t_{j}^{d}\right]$ is $b_{i} / \Delta t_{j}^{d}$. The total change-related penalty payments are given in (4).

$$
c_{j t}=\sum_{i} d_{i t} L_{i} \cdot\left(\begin{array}{l}
1-\max \left(0, \min \left(\frac{b_{i} \Delta t_{j}^{d}}{L_{j}^{s}}, 1\right)\right) \\
+\min \left(1, \frac{b_{i}}{\Delta t_{j}^{d}}\right)
\end{array}\right)
$$

\section{F. Volatile process demand}

When a $\mathrm{CAB}$ is faced with rather volatile demand for business processes, a finer-grained approach is required to determine the number of disrupted process instances, as the number of process instances requested in $t_{j}$ might deviate widely from the number of requests expected in precedent and subsequent timeslots. Hence, here the process demand levels in each timeslot and the position of $j$ 's execution in $i$ needs to be taken into account.

In scenarios without a queuing option, the number of change-related SLA violations can be estimated in the following way. Let $L_{i,(j-1)}$ be $i$ 's time required to execute all services preceding step $u_{i j}$ where $j$ is invoked. Consequently, $d_{i j t}^{p}$ equals $i$ 's total demand during $\left[\left(t_{j}-L_{i,(j-1)}\right),\left(t_{j}-\right.\right.$ $\left.L_{i,(j-1)}\right)+L_{j}^{s}$ ] (instances executing $j$ in $t_{j}$ ) and $i$ 's demand during $\left[\left(t_{j}-L_{i,(j-1)}-\Delta t_{j}^{d}\right),\left(t_{j}-L_{i,(j-1)}\right)\right]$ (instances invoking $j$ during $j$ 's outage). Hence, the total demand for $i$ that is penalized as a result of changing $j$ in $t, d_{i j t}^{p}$, is the demand during $\left[\left(t_{j}-L_{i,(j-1)}-\Delta t_{j}^{d}\right),\left(t_{j}-L_{i,(j-1)}\right)+L_{j}^{s}\right]$. By forecasting the aggregated demand during the period of time as mentioned above $\left(d_{i j t}^{p}\right)$, total penalty payments $c_{j t}$ can be estimated using (1).

If queuing is a valid option, as in our basic model, we need a fine-grained determination of $d_{i j t}^{p}$. To approximate changerelated downtime costs $c_{j t}$, again we forecast the process demand during the period given in (5) and substitute the term on the left-hand side in (4), $d_{i t} \cdot L_{i}$ by the resulting sum.

$$
\left[\left(t_{j}-L_{i,(j-1)}-\Delta t_{j}^{d}\right),\left(t_{j}-L_{i,(j-1)}\right)+L_{j}^{s}\right]
$$

\section{G. Probabilistic demand and downtime distribution}

So far, we used deterministic approximations for expected change-related downtime and demand levels per timeslot. In reality, these values are stochastic variables following certain probability distribution. Ignoring the probabilistic nature of demand and downtime might be acceptable for very narrow probability distributions, but otherwise this is not advisable as it may lead to inaccurate cost estimation and poor change scheduling decision making, as described later in this paper.

Suppose an RFC for service $j$ and a depending business process $i$ with high penalties to pay on execution time violation. Let the mean change-related downtime of $j$, as observed in the past, be 10, but varying broadly. Suppose there is a decision to make to either start the change at $t=0$ or at $t=50$. The demand for $i$ is expected to be slightly lower during $t=0-9$ than during $t=50-59$ but increases rapidly from $t=10$ onwards, while demand is expected to be of constant level after $t=59$. The deterministic model would certainly select $t=0$. A stochastic model explicitly taking into account uncertainty of downtime would select $t=50$, which would be the better decision as the risk of high change-related costs is reduced.
However, putting too much stochastic information into a mathematical model makes it intractable at least for medium and large problem sizes and limits its practical applicability. Hence, to consider the stochastic nature of the variables while keeping the calculation computable, we draw on a stochastic formulation with simple recourse, as introduced for example by de Boer [27] or Birge and Louveaux [28]. The principle is illustrated in Fig. 3 with normally distributed downtime. We

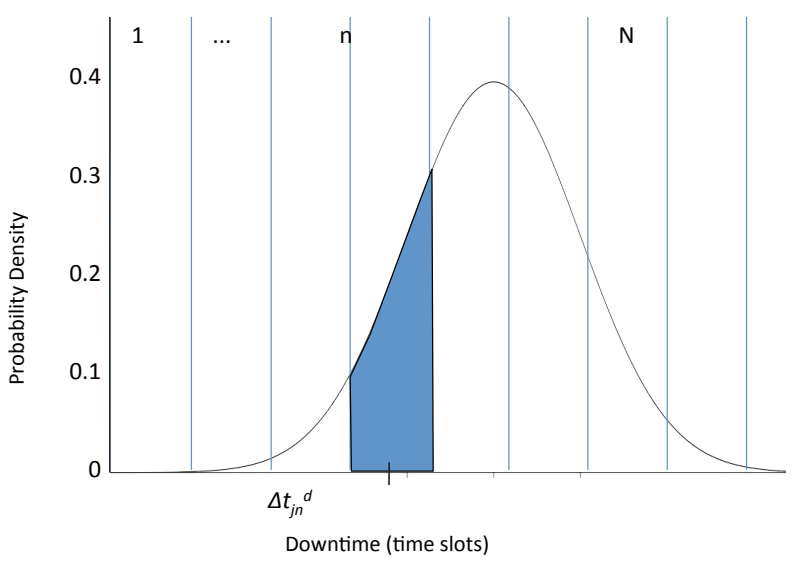

Fig. 3: Probabilistic Modeling of Downtime

separate the distribution into $N$ sequential discrete sections $n(n=1, \ldots, N)$. The cumulated probability of a section is then interpreted as one of $N$ downtime singletons, whereas we suppose downtime can only take these discrete values: $\Delta t_{j}^{d} \in\left\{\Delta t_{j 1}^{d}, \Delta t_{j 2}^{d}, \ldots, \Delta t_{j N}^{d}\right\}$ with certain probabilities $P\left(\Delta t_{j n}^{d}==\Delta t_{j}^{d}\right)$.

Using the formulas (1) and (4), or their extensions for volatile demand as described in the previous subsections, for each of the $N$ discrete downtime values $\Delta t_{j n}^{d}$, the expected costs $c_{j t n}$ of changing $j$ in $t$ can be determined. The expected costs are then derived as the sum of the weighted cost expectation, computed as given in (6).

$$
c_{j t}=\sum_{n} \sum_{i} c_{j t n} P\left(\Delta t_{j}^{d}==\Delta t_{j n}^{d}\right)
$$

The left part of the formula computes the costs of a change of $j$ in $t$ if the downtime would be exactly $\Delta t_{j n}^{d}$. The term on the right-hand side of the formula is a correction for the uncertainty (probability) in downtime: a weight. Likewise we model stochastic business process demand in a timeslot by calculating change-related costs for different possible demand levels, and weighting these demand levels with their associated probabilities.

For reasons of brevity, we refer below to the deterministic cost estimations in (2) and (4) even though all statements are valid for probabilistic cost estimations as well.

Please note that additional stochastic sources exist, like service execution times and - as a result - process execution times, which can be addressed in the same manner as stochastic downtime and demand. 


\section{H. Non-linear processes and multiple service invocations}

The determination of change-related penalties as introduced in the previous section assumes linear business processes and service flows with a predetermined sequence of service executions. In practice, business processes take different branches based on certain conditions and business process variations do exist. One branch might include a changed service while others do not. Hence, a finer-grained demand forecast is required for each possible branch, as ignoring conditional branches would overrate the number of expected penalty payments. By analyzing the demand history of executed branches, a differentiated forecast for each branch can be derived. Each branch can then be modeled as an individual process type.

Process definitions that involve the invocation of a service $j$ several times, for example in iterative sequences, cannot be demodulated in the same manner by defining each possible flow as a separate process, as such processes are more vulnerable to delay and disruption.

Reconsidering (2), in smooth demand scenarios we can estimate change-related costs by multiplying the costs derived by (2) by the number of occurrences of $j$ in a process $i$ definition if and only if downtime never exceeds the time lag between two $j$-occurrences. The same holds for costs in infrastructures with queuing options as given in (4). In the case of volatile demand, the interval where demand is aggregated as given in (5) needs to be extended. One additional interval per occurrence of $j$ (e.g., each loop iteration) in $i$ 's process definition needs to be included, where the term $L_{i,(j-1)}$ in (5) is adjusted for each occurrence of $j$ in $i$ 's definition.

If there is a certain probability that downtime overlaps with two or more $j$-occurrences, the procedure described in the previous paragraph needs to be modified in order to avoid double or triple counting of penalty costs per process instance. For reasons of brevity, we sketch the principle idea of a heuristic that we apply in that case without going into detail here. First, for each occurrence of $j$ in a process definition we estimate change-related costs in isolation (by ignoring other occurrences of $j$ ). Subsequently, we employ probabilities that downtime overlaps two or more particular occurrences of $j$ as weights for the costs per $j$-occurrence. Expected costs are then the sum of costs for each $j$-occurrence.

\section{CHANGE SCHEDULING DECISION MODEL}

In the next subsection, we will introduce a scheduling decision model for uncorrelated changes. Based on this model, extensions are proposed to consider other types of operational risks and costs associated with service transitions (i.e., changes). Subsequently, we address the handling of correlated changes. The models aim to find optimal change timeslots with minimum resulting business impact in terms of cost.

\section{A. Basic scheduling model}

We will now introduce mathematical programming models to determine the schedule for a set of uncorrelated changes with minimum expected overall service level violation costs.
We assume that a penalty is paid per SLA violation. In the previous chapter we estimated the costs of changing a service $j$ in a timeslot $t$.

Given these estimates, finding the optimal change schedule for uncorrelated changes is possible by scanning all timeslots and choosing the change timeslot with the lowest expected costs. To formalize the decision model, we introduce a binary decision variable $x_{j t}$ indicating whether $j$ 's change is started in $t$ or not. Let $J_{R F C}$ be the set all RFCs.

The objective function, aimed at minimizing the total sum of change-related penalty payments for all $i$-instances invoking $j$ is given in (7).

$$
\sum_{j \in J_{R F C}} \sum_{i: u_{i j}>0} \sum_{t}\left(c_{i j t} x_{j t}\right)
$$

We set the first timeslot of our change planning period to $t=0$ and assume to obtain $J_{R F C}$ before $t=0$. Please note that in practice some changes - in particular urgent changes - will be requested on a continuous time base rather than bundled. Means of addressing this are either to apply online scheduling algorithms or to recalculate the optimization problem each time a new RFC is submitted, or in a certain frequency (e.g., once an hour or once a day). In the literature, the second procedure is usually called optimization with rolling horizon. As we divide time into timeslots, time-related parameters are of positive integer type $\left(t_{j}, \Delta t_{j}^{d}, t_{j}^{*}, b_{i}, L_{i}, L_{j}^{s} \in Z_{0}^{+}\right)$.

Penalty, cost, and demand parameter are of positive real type $\left(d_{i t}, d_{i j t}, p_{i}, c_{i j t} \in R_{0}^{+}\right)$.

\section{B. Change deadlines and waiting costs}

As further constraints we introduce change implementation deadlines $t_{j}^{*}$. Depending on the severity of a change, there is generally an urgency associated with a change, defining a deadline when a change needs to be implemented and the service is again available. Considering a planned, predictable change-related downtime (e.g., a server reboot), this constraint can be formulated using (8).

$$
\sum_{t \leq t_{j}^{*}-\Delta t_{j}^{d}} x_{j t}=1, \forall j \in J_{R F C}
$$

Equation (8) defines it as mandatory to meet change deadlines. Considering the stochastic nature of change-related service downtime, depending on the underlying distribution it can no longer be guaranteed to meet deadlines and only a certain probability can be assigned to punctual change implementation. Therefore (8) is relaxed by claiming that a change must at least start before the change deadline, as given in (9).

$$
\sum_{t \leq t_{j}^{*}} x_{j t}=1, \forall j \in J_{R F C}
$$

Note that exceeding a deadline might entail a predefined penalty or extra payments for each additional timeslot that a service is down, either to repair the service in the case of unplanned downtime, or time to fulfill a change in the case of planned downtime. Hence, the later a change is started, the higher the expected costs of a deadline violation will be since 
the probability of completing change fulfillments before their deadline will decrease continuously.

Assuming a variable penalty model where $\alpha_{j}$ denotes the additional costs per timeslot $j$ 's change deadline is exceeded, (11) minimizes the expected overall deadline violation cost.

$$
\min \sum_{j \in J_{R F C}} \sum_{i: u_{i j}>0} \sum_{n} \sum_{t}\left(\begin{array}{l}
\alpha_{i} P\left(\Delta t_{j}^{d}==\Delta t_{j n}^{d}\right) . \\
\max \left(0, x_{j t} t+\Delta t_{j n}^{d}-t_{j}^{*}\right)
\end{array}\right)
$$

As in (6), we model the random variable downtime using a stochastic formulation with simple recourse. Again, the formula computes the deadline violation costs when starting a change of $j$ in $t$ if the downtime would be exactly $\Delta t_{j n}^{d}$, weighted by the probability that the realization of the downtime $\Delta t_{j}^{d}$ equals $\Delta t_{j n}^{d}$. To consider deadline violation cost in the scheduling model, formulation (11) needs to be added to the objective function in (7).

For reasons of clarity, in the following equations we only assume downtime to be random; demand or service execution times can be modeled stochastically as well, as shown earlier.

Furthermore, the moment an RFC is submitted, there may already be a need felt for the change to be implemented, as the business may suffer until this has been done. For example, this may be due to a service being unavailable, as would happen if the change request was initiated as a result of an incident, or there may be other negative impact causes, like lost opportunities such as would occur for a change meant to bring in a new required service. With $\gamma_{j}$ as the implicit costs of waiting one more timeslot, waiting costs are considered in the objective functions as formulated in (11).

$$
\min \left(\sum_{j \in J_{R F C}} \sum_{n} \sum_{t}\left(\begin{array}{l}
\gamma_{j} x_{j t}\left(t+\Delta t_{j n}^{d}\right) . \\
P\left(\Delta t_{j}^{d}==\Delta t_{j n^{d}}\right)
\end{array}\right)\right.
$$

\section{Change windows}

To mitigate the risk of downtime during operational working hours, change implementations are usually restricted to a set of change windows, e.g. weekends or nighttimes. Violating a change window restriction might have serious impacts on the business, as that means a critical service is down at times when this service is frequently requested. Therefore, penalties are to be paid for exceeding a change window. Let $T_{c j}$ be the set of change windows $w(w=1, \ldots, W)$, where each change window has an associated timeslot range of $\left[t_{j w}^{s t a r t}, t_{j w}^{e n d}\right]$. Again, considering downtime as a random variable, risk of change window violations increases the later a change is started in a change window. With $\beta_{j}$ quantifying the cost per timeslot a change window is exceeded for a change of service $j$, and the restriction that a change must (at least) start inside a change window $\left(t_{j} \in T_{c j}\right)$, the formula aimed at minimizing change windows violation costs is given in (12).

$$
\min \sum_{j \in J_{R F C}} \sum_{t}\left(\begin{array}{l}
\max \left(0, \beta_{j}\left(t x_{j t}+\Delta t_{j}^{d}\right)-\right. \\
\left.\min \left(t_{j w}^{\text {end }}: t_{j w}^{\text {end }}>t_{j}\right)\right)
\end{array}\right)
$$

Although (12) models downtime in a deterministic way, downtime modeled as a random variable can be done as in previous formulas.

\section{Dependent changes}

So far in our formulations we handle multiple independent changes. As changes might be correlated (e.g. multiple services must be changed in a mandatory order to re-engineer a whole business process), we now extend the model formulation to consider correlated or dependent changes. First, changes might be started in a certain sequence $\left(t_{j}<t_{(j+1)}\right)$, or a change must be implemented before the next change may be scheduled $\left(t_{j}+\Delta t_{j}^{d}<t_{(j+1)}\right)$. Here, the constraint in our scheduling model is $\left(t x_{i t}<t x_{(j+1) t}\right)$, or $\left(t x_{j t}+\Delta t_{j}^{d}<\right.$ $\left.t x_{(j+1) t}\right)$, respectively.

Besides a mandatory change scheduling order, changes might be statistically correlated, for example in terms of a reduction of aggregated downtime when executing changes together. Imagine two patches to a server operating system, both requiring a reboot. On the one hand, applying these patches together might reduce the overall change duration, as only one reboot is required. On the other hand, for example due to potential incompatibilities, installing both patches at once might result in higher risk in terms of higher downtime variance. Let $M\left(\Delta t_{j}^{d}\right)$ be the mean value of downtime resulting from changing $j$, and let $M\left(\Delta t_{(j+1)}^{d}\right)$ be $(j+1)$ 's mean change-related downtime, assuming that j's change is implemented before changing $(j+1)$. Furthermore, let $M\left(\Delta t_{j}^{d}, \Delta t_{(j+1)}^{d}\right)$ be the mean downtime of changing $j$ and $(j+1)$ together (likewise we proceed for variance $V)$. We consider two changes to $j$ and $(j+1)$ as correlated if $M\left(\Delta t_{j}^{d}\right)+M\left(\Delta t_{(j+1)}^{d}\right) \neq M\left(\Delta t_{j}^{d}, \Delta t_{(j+1)}^{d}\right)$ or $V\left(\Delta t_{j}^{d}\right)+V\left(\Delta t_{(j+1)}^{d}\right) \neq V\left(\Delta t_{j}^{d}, \Delta t_{(j+1)}^{d}\right.$.

We treat each change item combination with significant deviant aggregated statistical values as one single change. Whereas arbitrary statistical moments can be chosen, as an example we focused on mean and variance. The decision to make is to either schedule all statically dependent changes separately or to schedule the new aggregated change instead. If the question is to either change $j$ or $(j+1)$ separately or, alternatively, to implement the aggregated change $(j, j+1)$, the XOR constraint can be formulated as given in (13).

$$
\sum_{t} x_{j t}+x_{(j+1) t}+2 x_{(j, j+1)}=2
$$

The deadline for $(j, j+1)$ is then set to $\min \left(t_{j}^{*}, t_{(j+1)}^{*}\right)$.

\section{E. Example scheduling model formulation}

In the preceding subsections we formulated components or model parts, which will now be assembled to derive a full scheduling decision model. Depending on infrastructure assumption (with or without queuing), business process demand profiles, and the amount of stochastic information to be considered, different model variants with different parts are suitable. Hence, in (14) we will give an example scheduling model assuming smooth and predictive demand, a scenario with queuing, and stochastic change-related downtime modeled as a discrete random variable. Furthermore, we consider change window violation costs, change deadlines with costs per timeslot a deadline is exceeded, and two changes for 
service $j=2$ and $j=3$ in a mandatory change order.

$$
\begin{aligned}
& \min \sum_{j \in J_{R F C}} \sum_{t: u_{i j}>0} \sum_{n} \sum_{t}\left[x_{j t} P\left(\Delta t_{j}^{d}==\Delta t_{j n}^{d}\right) .\right. \\
& \left.\left(\begin{array}{l}
\left(p_{i} d_{i t}\left(L_{j}^{s} \Delta t_{j n}^{d}\right)\right)+ \\
\left(\max \left(0, \beta_{j}\left(t x_{j t}+\Delta t_{j n}^{d}-\min \left(t_{j w}^{e n d}: t_{j w}^{e n d}>t_{j}\right)\right)\right)\right)+ \\
\left(\max \left(0, \alpha_{i}\left(x_{j t} t+\Delta t_{j n}^{d}-t_{j}^{*}\right)\right)\right.
\end{array}\right)\right]
\end{aligned}
$$

$$
\begin{array}{ll}
s . t . & \\
\sum_{t \leq t_{j}^{*}} x_{j t}=1 & \forall j \in J_{R F C} \\
x_{j t} \in\{0,1\} & \forall j \in J_{R F C}, \forall t \leq T \\
t_{j}, \Delta t_{j}^{d}, \Delta t_{j n}^{d}, t_{j}^{*}, b_{i}, t_{j w}^{e n d} & \in Z_{0}^{+} \\
d_{i t}, d_{i j t}, p_{i}, c_{i j t}, c_{j t}, b_{i} & \in R^{+} \\
L_{i}, L_{j}^{s}, \beta_{j}, \alpha_{i} & \in R^{+} \\
n & \in\{1, \ldots, N\} \\
w & \in\{1, \ldots, W\} \\
t & \in\{1, \ldots, T\}
\end{array}
$$

The objective function minimizes the expected sum of overall costs for service delays or disruptions, change window violations, and exceeding change deadlines. The first constraint ensures that each requested change is initiated before its deadline, the second constraint ensures the mandatory change order, and the subsequent constraints restrict the scope of decision variables and model parameters.

\section{RESCHEDULING}

The decision models select change timeslots with the lowest expected overall costs based on business process demand forecasting, assumed service downtime distributions, and penalties defined in SLAs. In particular if uncertainty in future process demand is high, it is advisable to check and - if necessary - adjust a change schedule determined in $t=0$ before approaching a time interval $t_{j}$ (with $j \in J_{R F C}$ ). In that way, further knowledge of process demand levels and better demand predictions can be derived to reschedule the change start times. For example, if demand forecasting in $\left(t_{j}-50\right)$ predicts much higher business process demand than initially expected, there is a decision to make on whether to retain $t_{j}$ or to start the change later or earlier.

However, when deciding to start changes later, increasing delays or waiting costs and a higher probability of violating change deadline and change window restrictions has to be taken into account. In our simulations described later in this paper we recalculate the schedule if the business process demand forecasts computed earlier differed by more than $5 \%$ from the demand predicted in order to avoid unnecessary reschedules. Alternatively, one could reschedule at a predefined frequency or use online scheduling mechanisms instead of recalculating complete scheduling plans. Note that in particular when using online-scheduling mechanism, the impact of adjusting the change time of a service on the whole scheduling plan needs to be considered (in case of correlated changes).

When approaching $t_{j}$, besides considering more accurate near-term demand forecasting, we further refine our schedule by considering the states (progress) of currently active process instances. If in $\left(t_{j}-1\right)$ the percentage of running process instances currently executing service $j$ exceeds our expectations, the estimated change-related costs increase and we try to derive an alternative schedule with lower costs based on current cost expectations.

\section{Simulation}

In this section, we evaluate the proposed scheduling models in numerous discrete event simulations based on data provisioned by a telecommunication provider.

We simulate scenarios with two different DTP systems, varying, non-deterministic process demand behavior, and different probabilistic change-related downtime distributions. Benchmark criterion is total change-related cost when scheduling RFCs with the proposed deterministic model (DET) or the probabilistic model (PROB) instead of choosing an arbitrary change start timeslot at the beginning of a change window (random selection model (RAND)). Furthermore, we relate resulting costs to the lowest possible costs, determined by computing an optimal change schedule (OPTIMUM) ex post. OPTIMUM is determined by scanning the total solution space with knowledge of process demand and change-related downtime durations and then choosing the change start time with minimum associated costs.

The remainder of this section is structured as follows. First, the target DTP systems for our simulations are introduced. Subsequently, the simulation set-up is described. Finally, we report and discuss simulation outcomes.

\section{A. Target systems}

As our scenario we consider two DTP systems of a large telecommunications company. Data on system configuration as well as data on process and service dependencies is provisioned. Historical data on demand for processes and data on service execution times are available via log files.

The first system, SYSTEM_A, is an Enterprise Application Integration (EAI) system related to the management of the company's retail customer segment. This system runs mainly automated business processes like billing, order entry, order update, or customer data registration. Most of the processes are initiated by customers and are realized by executing transactions. Transactions consist of services running on distributed servers. The system runs 18 business processes involving 34 transactions, which execute 61 different atomic services. For example, a process of adding a new customer requires the execution of services like a credit check, the assignment of a mobile phone number, entries in the CRM system and the billing system, the activation of GPS services, and many more steps. In this system, a transaction is comprised of multiple atomic services and a business process is comprised of multiple transactions. The system exhibits a network of dependencies, as one single transaction type is called by up to 
four several business processes, and atomic service types are included in up to 15 transaction types.

The second system, SYSTEM_B, serves as integration backbone for business processes related to the management of products originally sold as prepaid packages. The system serves two major types of processes: order-entry processes initiated by customers and processes used by partner enterprises like tariff administration. The supported functionality includes for example customer subscription and un-subscription, SIM and phone number handling, and age and status verification. The complexity of SYSTEM_B in terms of the number of processes, services, and interdependencies is comparable to the complexity of SYSTEM_A.

In both DTP systems, business processes have stringent SLAs regarding availability and maximum execution time. Besides On-Line Transaction Processing (OLTP) processes, typically requested and processed during daytime, long-running batch processes (for example churn calculations) are executed during nighttime with less stringent SLA-requirements. However, OLTP jobs are often executed during nighttime or at weekends as well. A high number of changes to the process and service infrastructure is made on a weekly basis; major infrastructure releases are made every on a monthly basis, including a change planning and implementation period.

\section{B. Simulation model}

We set the duration of each simulation run to 2,016 timeslots $t(t=0, \ldots, 2015)$ of 15 -minute durations, i.e., three weeks in total. For each timeslot, process demand is represented as the aggregated demand observed over a time span of 15 minutes.

We set the change windows to weekends (Friday 8pm Monday 8am) and for weekdays to nighttimes (from 8pm to $8 \mathrm{am}$ ). Change-related downtime during operational hours (i.e., a change window violation) is penalized according to (10). By setting $t_{j}^{*}=2015 \forall j \in J_{R F C}$ we ensure that all changes are implemented within a simulation run. Waiting costs are not considered. For OLAP processes we set the maximum execution time to three timeslots, and for batch jobs to seven timeslots. All SLA violations - including process disruption if queuing is not supported - are equally penalized by one monetary unit.

With demand logs covering a period of nine months in total, we define four different demand scenarios per DTP system. To characterize demand profiles including typical peak sizes and daily and weekly patterns used in our demand-forecasting model, we analyze demand logs over a period of two months for both DTP systems as training data. Based on these profiles we derive representative demand curves (in the probabilistic model, we generate five curves of ascending demand levels together with their corresponding probabilities). Simulations are then conducted with the real demand curves of three subsequent weeks. For the models, these curves are not known beforehand but need to be forecasted.

Additionally, in order to perform sensitivity analysis, we adjust the curves systematically to obtain different patterns with varying volatility in terms of peak to mean ratios and average peak lengths. Furthermore, for each obtained profile we increase demand uncertainty by adjusting different weeks of the training data differently. During a timeslot we generate demand that is equally distributed over an the timeslot length and adapt the demand level after each interval according to the simulated demand profile.

We classified the resulting demand scenarios into three categories, with 60 demand profiles per category:

- C1: Smooth and easily-predictable demand

- $C 2$ : Moderately volatile demand where peaks do not vary by more than $50 \%$ and demand burst-sizes do not exceed burst sizes in the training data

- C3: Volatile, uncertain demand with frequent, irregular, and high demand peaks (up to twice as high as peaks in the training data)

An example demand scenario is given in Fig. 4. The graph shows the demand profiles of four business processes depending on a changed service in a selected simulation scenario. In the scenario, weekly business process demand patterns are rather regular and predictable, as peak sizes and durations do not vary by more than $15 \%$ (C1 demand). For conducting

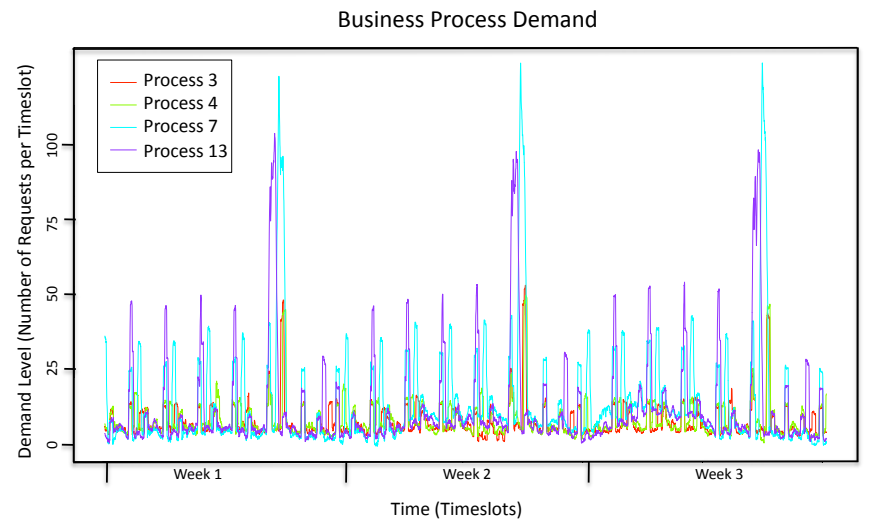

Fig. 4: Example Process Demand Scenario

the simulations, we arranged a list of 150 entries per DTP scenario, where the first 50 entries in the list represent RFCs for one service, the second 50 entries represent RFCs for two services, and the last 50 entries represent RFCs for three services. Services to be changed are arbitrarily selected from $J$, the set of all composite services. When two or more RFCs are required, we assume a mandatory change order, i.e., the first change must be implemented before the second change starts. We simulate each demand scenario for each entry in the list and repeat each simulation with change-related downtime following a specific probability mass function until results are significant.

Unfortunately, only rough statistics of change-related downtime are available at this stage. Hence, we approximated service downtime distributions based on insight from expert panels and based on some available data on downtime.

Service downtime is modeled as a discrete random variable, following one of two probability distributions: unexpected and expected downtime distribution.

For expected downtime (e.g., a necessary server reboot after installing an OS patch), we assume three possible symmetric 
Binomial distributions (PLANNED_1 - PLANNED_3) with a mean of three, four, or five timeslots with increasing variance, where downtime singletons could take values out of $[0,6]$, $[0,8]$, or $[0,10]$ time intervals. As an example with a mean downtime of three time intervals, and singletons in $[0,6]$, on the right-hand side of Fig. 5 the $\operatorname{Binomial}(6,0.5)$ probability distribution is depicted.

For unexpected downtime we consider a fat tail probability distribution as no change-related downtime usually occurs, except in some cases where then time is required for reparation. We use three Zipf-Mandelbrot distributions (Unplanned_1 Unplanned_3) with different variance, as we parameterize the distributions with population sizes of seven, nine or eleven timeslots, a power exponent of three, and zero shifts (the leftmost singleton is set to zero instead of one). On the lefthand side of Fig. 5, an unexpected downtime distribution with a population size of seven (0-6 timeslots), a power exponent of three and zero shift is depicted.

DET is parameterized with the median downtime and with mean demand per timeslot, while PROB minimizes the weighted expected outcome for all possible downtime durations for ten possible demand scenarios. According to
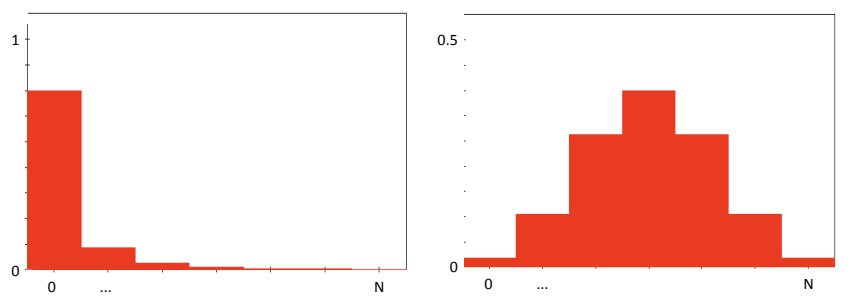

Fig. 5: Planned versus Unplanned Downtime Probability

the available log-files, service execution times vary only inbetween tight boundaries and in large set of preliminary simulations, solution qualities of the models did not significantly differ whether execution times were modeled as random variables or deterministic values. Hence, to keep the models computationally tractable, we model service execution times as deterministic by taking their means. Please note that the validity of this proceeding needs to be re-checked when considering target systems with a higher variance in service execution times.

Within one simulation run, for all changes we assume equal downtime probability distributions.

Although queuing is used in the company's DTP system, we repeat all simulations without a queuing option.

To configure, run, and analyze simulations, a tool has been developed (see Fig. 6). The figure shows a screenshot of a scenario with three business processes (composed of up to five transactions) that depend on a changed service. The figure shows two linear processes and a process where only some instances depend on a changed service.

\section{Simulator validation}

To ensure that the computerized simulation model possesses satisfactory accuracy consistent with the target system, we

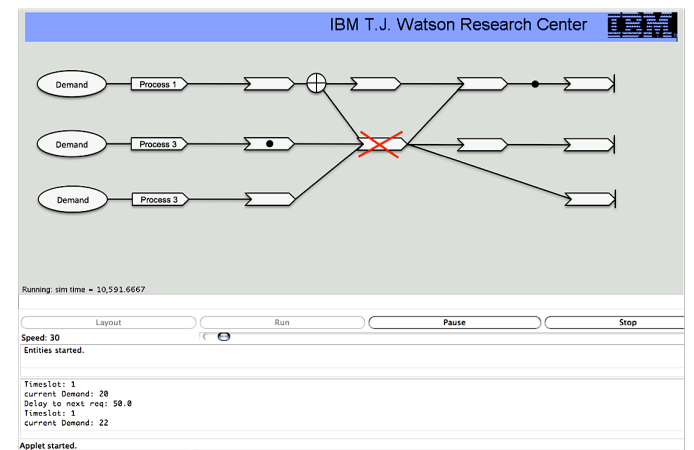

Fig. 6: Simulation Tool Screenshot

mainly draw on historical data validation as well as internal validation. Historical data validation was conducted by driving the simulation model with empirical traces provisioned by the telecommunications provider to ensure the simulator behaves as the real system behaves.

Internal validity was tested by conducting several replications of each simulation to determine the amount of stochastic variability in order to determine confidence intervals, as shown in section VI-D.

Data for system configuration, process landscape, service dependencies, as well as process demand curves and service execution times was obtained from real-world systems. However, as only small amounts of empirical data on downtime are available we consider downtime distributions based on that data and interviews with the company's IT service managers. As will be shown in the next subsection, the sensitivity of PROB's solution quality regarding downtime Data for system configuration, process landscape, service dependencies, as well as process demand curves and service execution times was obtained from real-world systems.

However, as only small amounts of empirical data on downtime are available we consider downtime distributions based on that data and interviews with the company's IT service managers. As will be shown in the next subsection, the sensitivity of PROB's solution quality regarding downtime distribution variation was moderate, at least for the distribution types and parameter variations applied in our simulations.

\section{Simulation Results}

The aggregated simulation results are presented in Table 1. The table shows average solution qualities of the scheduling models including $95 \%$ confidence intervals. Solution quality is expressed as a ratio of change-related costs derived by applying an OPTIMUM schedule divided by cost when scheduling changes using RAND, DET, or PROB. For example, if in a simulation run the change-related cost was 2 with an OPTIMUM schedule, and cost was 3 when applying DET, DET's solution quality ratio is $2 / 3$. Obviously, solution quality of a scheduling model increases with ratios approaching to 1 . The first row in Table 1 shows the average solution quality of the three models for all simulations, while rows 2-3 show ratios for samples using one of the two DTP systems only. On average, the solution quality ratio achieved by scheduling 
TABLE I: Aggregated Simulation Results

\begin{tabular}{|c|l|c|c|c|}
\hline \multirow{2}{*}{ ID } & \multirow{2}{*}{ SAMPLE } & \multicolumn{3}{|l|}{ SOLUTION QUALITY: MEDIAN (CONFIDENCE INTERVAL) } \\
\cline { 3 - 5 } & & PROB & DET & RAND \\
\hline \hline 1 & Total & $0.94(0.91,0.95)$ & $0.85(0.77,0.89)$ & $0.45(0.32,0.64)$ \\
\hline 2 & SYSTEM_A & $0.94(0.90,0.96)$ & $0.85(0.74,0.90)$ & $0.45(0.28,0.64)$ \\
\hline 3 & SYSTEM_B & $0.94(0.90,0.95)$ & $0.85(0.75,0.90)$ & $0.45(0.27,0.64)$ \\
\hline 4 & C1 Demand & $0.98(0.94,0.99)$ & $0.97(0.95,0.99)$ & $0.46(0.27,0.65)$ \\
\hline 5 & C2 Demand & $0.95(0.92,0.98)$ & $0.88(0.90,0.98)$ & $0.45(0.26,0.67)$ \\
\hline 6 & C3 Demand & $0.90(0.84,0.96)$ & $0.73(0.60,0.90)$ & $0.44(0.25,0.65)$ \\
\hline 7 & Queuing & $0.94(0.91,0.95)$ & $0.85(0.77,0.90)$ & $0.45(0.29,0.64)$ \\
\hline 8 & No Queuing & $0.94(0.90,0.94)$ & $0.84(0.75,0.89)$ & $0.45(0.27,0.62)$ \\
\hline 9 & Planned & $0.93(0.90,0.95)$ & $0.86(0.77,0.89)$ & $0.47(0.33,0.68)$ \\
\hline 10 & Unplanned & $0.95(0.91,0.96)$ & $0.83(0.77,0.89)$ & $0.41(0.32,0.67)$ \\
\hline 11 & 1 RFC & $0.95(0.93,0.97)$ & $0.85(0.81,0.88)$ & $0.45(0.29,0.64)$ \\
\hline 12 & 2 RFCs & $0.95(0.92,0.97)$ & $0.84(0.80,0.88)$ & $0.45(0.28,0.64)$ \\
\hline 13 & 3 RFCs & $0.94(0.92,0.96)$ & $0.84(0.76,0.87)$ & $0.45(0.28,0.65)$ \\
\hline
\end{tabular}

using PROB was 0.94, 0.85 for DET and 0.45 for RAND. The $95 \%$ confidence intervals indicate that solution quality of PROB is more stable than solution quality of DET or RAND.

Solution qualities did not differ significantly in samples with only SYSTEM_A or only SYSTEM_B. The broader confidence intervals in samples with only one particular system results from the bisect sample sizes.

As shown in rows 4-6 in Table 1, the average solution quality differed significantly between samples with different demand classes. In each demand class, PROB dominated DET (which again dominated RAND). However, the solution quality of PROB and DET compared to OPTIMUM as well as the dominance over RAND decreased with increasing demand uncertainty and volatility.

The aggregated effect of a queuing option on the solution quality can be derived by comparing row 7 (sample with a queuing option) with row 8 (sample without a queuing option). PROB, DET, and RAND performed only slightly better in scenarios with queuing.

The aggregated results in rows 9 and 10 show that the dominance of PROB over DET and RAND was higher in scenarios with unexpected downtime distributions, while solution quality of DET and RAND did not show significant dependence on the type of the downtime distribution.

Finally, rows 11-13 show separate results for samples with one, two, and three RFCs. PROB dominated DET and RAND in all three samples. DET and PROB both achieved highest solution quality in scenarios with one RFC, and the lowest solution quality in samples with three RFCs. RAND's solution quality did not decrease significantly in samples with one, two, or three RFCs.

Rescheduling changes by both the PROB and DET models was done in less than $2 \%$ of simulations with class 1 demands. In the sample with class 2 demand, DET rescheduled in over $80 \%$ of cases; PROB rescheduled in around $65 \%$ of cases. In simulations with class 3 demand, rescheduling was almost always done with DET, and in around 95\% of cases with PROB. By conducting ex-post analysis, we found out that rescheduling decreased costs in $96 \%$ of cases.

\section{E. Discussion}

In over 99\% (98\%) of simulations, scheduling with PROB (DET) resulted in lower change-related downtime costs compared to randomly selecting a change time interval. Hence, unsurprisingly, enterprises can benefit from scheduling (and rescheduling) changes considering the risk of change-related downtime as potential business impact can be reduced. As PROB dominated DET in almost all cases, we will focus our discussion on PROB.

We now analyze what we can say of PROB's solution quality and its sensitivity regarding different treatments. While PROB's overall solution quality was on average 0.94 , PROB achieved the highest solution quality in samples with $\mathrm{C} 1$ demand, with queuing, unplanned downtime, and only one change to implement. Here PROB's ratio was above 0.98 with a narrow $95 \%$ confidence interval $(0.96,0.99)$. We did not find significant differences between PROB's performance with these types of samples when using SYSTEM_1 or SYSTEM $\_$.

Broadly speaking, having reliable knowledge of downtime probability distributions and demand profiles, PROB is a very promising scheduling model for practitioners given the characteristics of an application scenario as mentioned above. When demand became more stochastic, the probabilistic model PROB performed significantly better than the deterministic model DET. As shown in Table 1, PROB's average solution quality decreased with increasing demand uncertainty and volatility as a consequence of forecasting inaccuracy and corresponding suboptimal change schedules. However, rescheduling mitigated the negative impact of imperfect knowledge of future demand to a great extent, as shown in Table 2.

PROB's solution quality in $\mathrm{C} 1$ demand scenarios was, on average, over 0.97 , whether schedules were refined or not. The solution quality difference here is low, as schedules almost never needed to be refined, since demand forecasts were of high accuracy and scheduling plans performed well. With increasing demand uncertainty, the solution quality of PROB without rescheduling during runtime decreased significantly from 0.97 in $\mathrm{C} 1$ demand scenarios down to 0.64 in $\mathrm{C} 3$ demand scenarios. Refining schedules over time increased the solution quality ratio in $\mathrm{C} 2$ demand scenarios by 0.05 to 0.95 , and in $\mathrm{C} 3$ demand scenarios by over 0.25 to a value of 0.90 . Compared to randomly selecting change timeslots in $\mathrm{C} 3$ demand scenarios, with a ratio of around 0.44, PROB significantly lowered costs even when faced with high demand uncertainty and volatility. In contrast to DET, PROB's solution quality did not

TABLE II: Impact of Rescheduling

\begin{tabular}{|c|l|c|c|}
\hline \multirow{2}{*}{ ID } & \multirow{2}{*}{ SAMPLE } & \multicolumn{2}{|c|}{ SOLUTION QUALITY: MEDIAN (CONFIDENCE INTERVAL) } \\
\cline { 3 - 4 } & & No RESCHEDULING & RESCHEDULING \\
\hline \hline 1 & C1 Demand & $0.97(0.95,0.99)$ & $0.98(0.96,0.99)$ \\
\hline 2 & C2 Demand & $0.90(0.82,0.93)$ & $0.95(0.92,0.98)$ \\
\hline 3 & C3 Demand & $0.64(0.55,0.72)$ & $0.90(0.84,0.96)$ \\
\hline 4 & Total & $0.84(0.78,0.88)$ & $0.94(0.91,0.97)$ \\
\hline
\end{tabular}

differ significantly in samples with unexpected and expected downtime distributions. Table 3 shows the average solution 
quality of RAND, DET, and PROB in samples with only expected downtime distributions with increasing variance from PLANNED_1 to PLANNED_3 (lines 1-3). Lines 4-6 show the average solution quality of the models in simulations with unexpected downtime distributions, with increasing variance from UNPLANNED_1 to UNPLANNED_3.

As can be seen, PROB dominated DET and RAND in all samples independently of downtime distributions. As discussed in section III.G, deterministic models perform worse when probability distributions are broadened and effects do not scale linearly with realizations of a random variable. Correspondingly, DET's solution quality decreased by 0.026 when comparing the sample PLANNED_1 and PLANNED_2, while PROB's average solution quality decreases by 0.006 only likewise when comparing PLANNED_2 and PLANNED_3. In samples with unplanned downtime distributions, the decrease ratio was around 0.045 for DET and 0.01 for PROB. In general, although better performance is expected when

TABLE III: Impact of Downtime Distributions

\begin{tabular}{|c|l|c|c|c|}
\hline \multirow{2}{*}{ ID } & \multirow{2}{*}{ SAMPLE } & \multicolumn{3}{|l|}{ SOLUTION QUALIT: MEDIAN (CONFIDENCE INTERVAL) } \\
\cline { 3 - 5 } & & PROB & DET & RAND \\
\hline \hline 1 & PLANNED_1 & $0.95(0.92,0.96)$ & $0.87(0.77,0.89)$ & $0.45(0.31,0.62)$ \\
\hline 2 & PLANNED_2 & $0.95(0.91,0.96)$ & $0.85(0.76,0.89)$ & $0.45(0.29,0.63)$ \\
\hline 3 & PLANNED_3 & $0.94(0.91,0.96)$ & $0.82(0.72,0.89)$ & $0.44(0.29,0.64)$ \\
\hline 4 & UNPLANNED_1 & $0.96(0.93,0.97)$ & $0.87(0.79,0.88)$ & $0.46(0.32,0.64)$ \\
\hline 5 & UNPLANNED_2 & $0.95(0.91,0.97)$ & $0.85(0.77,0.88)$ & $0.45(0.30,0.65)$ \\
\hline 6 & UNPLANNED_3 & $0.94(0.90,0.97)$ & $0.83(0.71,0.86)$ & $0.44(0.30,0.64)$ \\
\hline
\end{tabular}

stochastic influences are considered, often the benefits are low and stochastic modeling is not worth the effort; a wellknown example is seat inventory control in airline network revenue management. Based on the results of our simulations, in the case of the business impact of change-related downtime, probabilistic modeling does significantly lower the costs.

Comparing PROB's solution quality in samples with one, two, or three RFCs, PROB's simulation results did not exhibit significant differences for different numbers of RFCs in $\mathrm{C} 1$ or $\mathrm{C} 2$ demand scenarios. In C3 scenarios, PROB's solution quality decreased (although moderately) with the number of RFCs to fulfill, as inaccuracies in demand forecasting impacts the solution quality several times.

\section{F. Handling the computational complexity}

The scheduling decision problem as formulated in our work is proven to be strongly NP-hard. Hence, the problem is only computationally tractable for problem instances up to a certain size, in particular as the complexity grows with $T, I, J, K, N, W$, and $J_{R F C}$.

However, for uncorrelated changes we determined the change-related costs of changing a service $j$ in time interval $t$ by only considering timeslots which could potentially be affected by a service's downtime (i.e., the range of the downtime random variable). For this reason we were able to efficiently parallelize the computation to 16 standard Intel Dual Core $2.4 \mathrm{MHz}$ computers and run one simulation within less than 30 seconds. For simulations with a mandatory change order we first divided the simulation time period into 16 sub-periods, where each computer was instructed to choose a timeslot within a particular sub-period as change timeslot for the first change and to solve the problem with this restriction. Choosing the result with the lowest expected cost then derived the solution. Simulations with three changes in a mandatory change order are run within a period of four minutes.

However, considering broader downtime distributions has a huge impact on the computational time required to solve a problem. For example, simulations assuming unplanned downtime distribution with a range of 100 timeslots required around 26 minutes for uncorrelated changes, and around three hours for correlated changes. Therefore, to solve problem instances with larger sets $J_{R F C}$, in particular with correlated changes to implement, and broader change-related downtime distributions, the amount of considered stochastic information can be reduced, timeslots may be coarsened, and heuristics may be applied, for example to pre-select promising change timeslot candidates.

\section{SUMMARY AND OUTLOOK}

In this paper we introduced a probabilistic model for analyzing the business impact of changes in a network of services. We analyzed change-related operational risks to active business process instances and techniques to relate these risks to financial metrics. Based on the analytical model, we developed a decision model to schedule service changes in a way to reduce total expected change-related costs.

In extensive sets of simulations based on industry data, we evaluated the efficiency of the model in various scenarios with two different DTP systems, different downtime probability distributions, different numbers of services to be changed, and uncertain process demand behavior. Simulation outcomes indicate that the proposed model schedules changes with resulting costs approaching the minimum costs theoretically possible if future process demand is easily-predictable and change-related downtime distributions are known; by refining scheduling plans over time, the scheduling model still achieved significant cost savings even in scenarios with highly volatile demand that was hard to predict. Although the solution quality decreased with demand uncertainty and the number of RFCs, the sensitivity was moderate. In no sample did PROB's costs exceed the minimum costs by more than $11 \%$, which indicates robustness. This robustness should make the scheduling model a useful tool in supporting practitioners in their decision making of how to schedule changes.

To the best of our knowledge, our work is the first to formally quantify the risk of changing services to the business (processes), or to derive decision models that allow organizations to schedule service changes with minimum total expected business impact.

In order to make the model usable for practitioners to support their change-scheduling decision making, besides process demand forecasting, which can usually be done with sufficient accuracy, reliable estimators for change-related downtime distributions are required to parameterize the models. This information is usually not available to hand in organizations and 
first need to be collected based on empirical observation. In target systems with more variable service execution times than in the studied DTP systems, the sensitivity of PROB's solution quality to the variance must be analyzed prior to applying it in such scenarios. In particular in semi-automated processes with human working tasks such as interactive workflows, the execution times of services usually vary broadly. Reliable data needs to be collected in order to estimate probability distributions of service execution times.

In the future, we plan to apply the models as a decision support tool for change scheduling in selected businesses. Future working plans also include more exhaustive sets of simulations including manual or semi-automated business processes with stochastic service execution times. To keep the model computationally tractable and therefore applicable in practice for larger scenarios, we will work on meta-heuristics and change timeslot pre-selection algorithms.

\section{REFERENCES}

[1] J. P. Sauve, R. A. Santos, R. R. Almeida, and J. A. B. Moura, "On the risk exposure and priority determination of changes in it service management," in Managing Virtualization of Networks and Services. Berlin/Heidelberg: Springer, 2007, vol. 4785, pp. 147-158.

[2] OGC, Service Support (It Infrastructure Library Series). London, UK: The Stationery Office, 2000.

[3] "The bottom line project. it change management challenges - results of 2006 web survey," Computing Systems Department, Federal University of Campina Grande, Tech. Rep. DSC005-06, 2006.

[4] OGC, "Itil change management maturity benchmark study," Evergreen Consulting, Tech. Rep., 2006.

[5] IBM, "Tivoli change and configuration management database," 2009, [Online]. Available: http://www.ibm.com/software/tivoli/products/ccmdb/.

[6] O. Huehn and C. Markl, "Performance modeling for sla-compliant but cost-effective it-service provisioning," in Proc. 17th Workshop on Information Technology and Systems (WITS), Montreal, Canada, 2007.

[7] T. Setzer, K. Bhattacharya, and H. Ludwig, "Decision support for service transition management," in Proc. 11th IEEE Network Operations and Management Symposium (NOMS), Salvador, Brazil, 2008.

[8] OGC, Management of Risk, Guidance for Practioners. London, UK: The Stationery Office, 2007.

[9] S. Kaplan and B. J. Garrick, "On the quantitative definition of risk," in Risk Analysis, 1981, vol. 1, pp. 11-27.

[10] OGC, "Official itil website," 2009, [Online]. Available: http://www.bestmanagement-practice.com/IT-Service-Management-ITIL.

[11] IT-Governance-Institute, COBIT 4.1. Isaca, 2007.

[12] IBM, "It service management - change and configuration management: Reducing risk by understanding your infrastructure." 2008, [Online]. Available: http://www-03.ibm.com/solutions/tsolutions/doc/.

[13] Cisco, "Business management case study: How cisco it uses software configuration management to minimize business," 2007, [Online]. Available: http://www.cisco.com/web/about/ ciscoitatwork/casestudies/business_management_dl2.html.

[14] BMC, "Bmc remedy change management dashboard," 2009, [Online]. Available: http://www.bmc.com/products/documents/25/43/62543/62543.pdf.

[15] Hewlett-Packard, "Hp release control software," 2009, [Online]. Available: https://h10078.www1.hp.com.

[16] M. Pinedo, Scheduling: Theory, Algorithms, and Systems. Prentice Hall, 2001.

[17] E. Demeulemeester and W. Herroelen, "A branch-and-bound procedure for the generalized resource-constrained project scheduling problem," Operations Research, vol. 45, no. 2, p. 201212, 1997.

[18] A. Keller, J. Hellerstein, J. Wolf, K.-L. Wu, and V. Krishnan, "The champs system: change management with planning and scheduling," in Proc. IEEE Network Operations and Management Symposium (NOMS), Seoul, Korea, 2004.

[19] J. Goolsbey, "Risk-based it change management,," Pacific Northwest National Laboratory - IT Services Division, Tech. Rep., 2007.
[20] R. Rebouas, J. Sauv, A. Moura, C. Bartolini, and D. Trastour, "A decision support tool for optimizing scheduling of it changes," in Proc. 10th IFIP/IEEE Symposium on Integrated Management, Munich, Germany, 2007.

[21] D. Trastour, M. Rahmouni, and C. Bartolini, "Activity-based scheduling of it changes," in Inter-Domain Management - Lecture Notes in Computer Science. Berlin/Heidelberg, Germany: Springer, 2007, vol. 4543/2007, pp. 73-84.

[22] J. Sauve, R. Santos, R. Reboucas, A. Moura, and C. Bartolini, "Change priority determination in it service management based on risk exposure," IEEE Transactions on Network and Service Management, vol. 5, no. 3 , pp. 178-187, 2008.

[23] R. Kembel and R. Cummings, "Fibre channel: A comprehensive introduction," Northwest Learning Association, Tech. Rep., 2007.

[24] J. Walsh, A. Rockall, and A. Sudarsky, "Method and apparatus to estimate downtime and cost of downtime in an information technology infrastructure," U.S. Patent 20030187967, March 2002.

[25] D. A. Patterson, "A simple way to estimate the cost of downtime," in Proc. 16th System Administration Conference LISA, Philadelphia, USA, 2002.

[26] "How to calculate cost of downtime," Silverton Consulting, Tech. Rep., 2007, [Online]. Available: email: info@ silvertonconsulting.com.

[27] S. V. d. Boer, R. Freling, and N. Piersma, "Stochastic programming for multiple-leg network revenue management," ORTEC Consultants, Tech. Rep., 1999.

[28] J. R. Birge and F. Louveaux, Introduction to Stochastic Programming. New York, USA: Springer, 1997

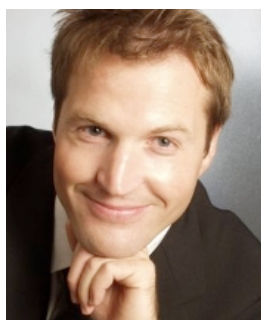

Thomas Setzer received his Masters degree in Business Engineering from the University of Karlsruhe, Germany, and his Ph.D. degree in Information Systems from the Technische Universität München (TUM), Germany. He worked as an engineer for the European Parliament and Cinebank Media. He is currently working at TUM as assistant professor on IT Management topics, in particular data center virtualization and automation. Thomas won the INFORMS ISS Design Science Award 2008 and his work is funded by the German Research Foundation.

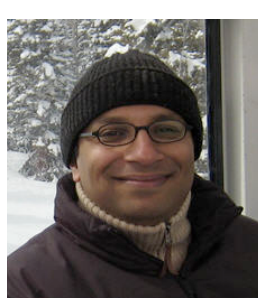

Kamal Bhattacharya graduated with a $\mathrm{PhD}$ in Theoretical Physics from Göttingen University, Germany. Currently Kamal manages a team at IBM Research exploring new ideas in the area of IT Optimization. His research focuses on topics around data center consolidation, application migration, and business value driven strategies for IT Service Management. Prior to joining IBM Research he has been working as an IT architect at IBM Global Services and received two Outstanding Tech. Achievement awards and several IBM Research Division awards.

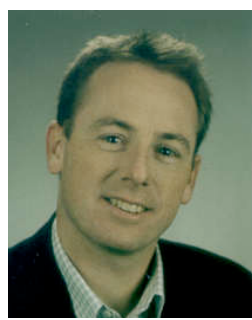

Heiko Ludwig received his Masters and $\mathrm{PhD}$ degrees from the University of Bamberg, Germany. $\mathrm{He}$ is a Research Staff Member at IBMs T.J. Watson Research Center. He is working on issues of service management and service platforms, primarily managing large-scale, loosely-coupled, crossdomain distributed systems, including service outsourcing and cloud computing. He has published numerous papers and articles and serves as editor and conference organizer in various capacities. 